\title{
International Cartography Master graduates starting their career
}

\author{
Juliane Cron ${ }^{\text {a, }}$, Liqiu Meng a ${ }^{\text {a }}$ Edyta P. Bogucka ${ }^{a}$, Georg Gartner ${ }^{b}$, Dirk Burghardt ${ }^{c}$, Menno-Jan \\ Kraak ${ }^{\mathrm{d}}$, Corné van Elzakker ${ }^{\mathrm{d}}$, Britta Ricker ${ }^{\mathrm{d}}$ \\ a Technical University of Munich, Chair of Cartography, juliane.cron@tum.de, liqiu.meng@tum.de, e.p.bogucka@tum.de \\ ${ }^{b}$ Vienna University of Technology, Research Group Cartography, georg.gartner@tuwien.ac.at \\ ${ }^{c}$ Technische Universität Dresden, Institute of Cartography, dirk.burghardt@tu-dresden.de \\ ${ }^{d}$ University of Twente/ITC, Department of Geo-InformationProcessing,m.j.kraak@utwente.nl, c.vanelzakker@utwente.nl, \\ b.a.ricker@utwente.nl \\ * Corresponding author
}

\begin{abstract}
:
Since its commencement eight years ago, the Cartography Master program has enrolled 155 students in eight annual intakes from 58 different countries. In this paper we share the experiences of the alumni of the first 5 intakes of the Cartography M.Sc. program that were gained via an online survey. Through the results of the survey we are able to answer the following questions: In which fields of work are our alumni getting involved? What kinds of businesses employ cartography alumni? In which countries are the graduates working? Furthermore, we would like to introduce some of our alumni: What are they doing now, how have they applied the things they learned during their studies in their careers and what message do they have for our future students? What do they consider as their most important achievements since graduation? What did they take with them from studying at four different universities? And how do they see their future?
\end{abstract}

Keywords: Cartography M.Sc., cartographic education, cartographic competences, alumni survey, career paths

\section{What is the Cartography Master program?}

The international Master of Science in Cartography (Cartography M.Sc., www.cartographymaster.eu) was launched in 2011 and is a joint cooperation of four universities: Technical University of Munich (TUM) in Germany, Vienna University of Technology (TUW) in Austria, Technische Universität Dresden (TUD) in Germany and the University of Twente (UT) in the Netherlands. The Cartography program intents to set innovative standards in international cartographic education and research and develops academic and cultural exchange. With its unique curriculum, the Master's program aims to educate students to become the best future engineers in cartography and to prepare them either for a career in the scientific or in the commercial field of cartography. Graduates are prepared to develop cartographic tools to analyse and visualise our rapidly changing world. The cooperation of four universities intents to give the graduates an access to an international network and an optimal start into a cartographic career. Job possibilities are ensured not only in their home countries, but also worldwide. Due to the value of cartography and uniqueness of the program, the Education, Audiovisual and Culture Executive Agency of the European Union has awarded co-funding for student scholarships two times in a row within the Erasmus+ Erasmus Mundus funding scheme. But over the years we see a steady increase of participants without a scholarship.

\section{Who are our students?}

The mission of the program is to attract students who are self-disciplined and show initiative, are independent thinkers, but also team players and are interested in art, science and technology. Candidates are eligible to be admitted to the Cartography program if they have obtained a Bachelor degree or equivalent in a discipline related to cartography, geo-information, geography, geodesy, geology or computer science. As the program is completely taught in English, students need to prove their language proficiency. The final selection of students is also based on their personal motivation and letters of recommendation.

Approximately $40 \%$ of the students originate from Europe, $40 \%$ from Asia, $10 \%$ from North America, $8 \%$ from Africa and $3 \%$ from South America (Figure 1). 


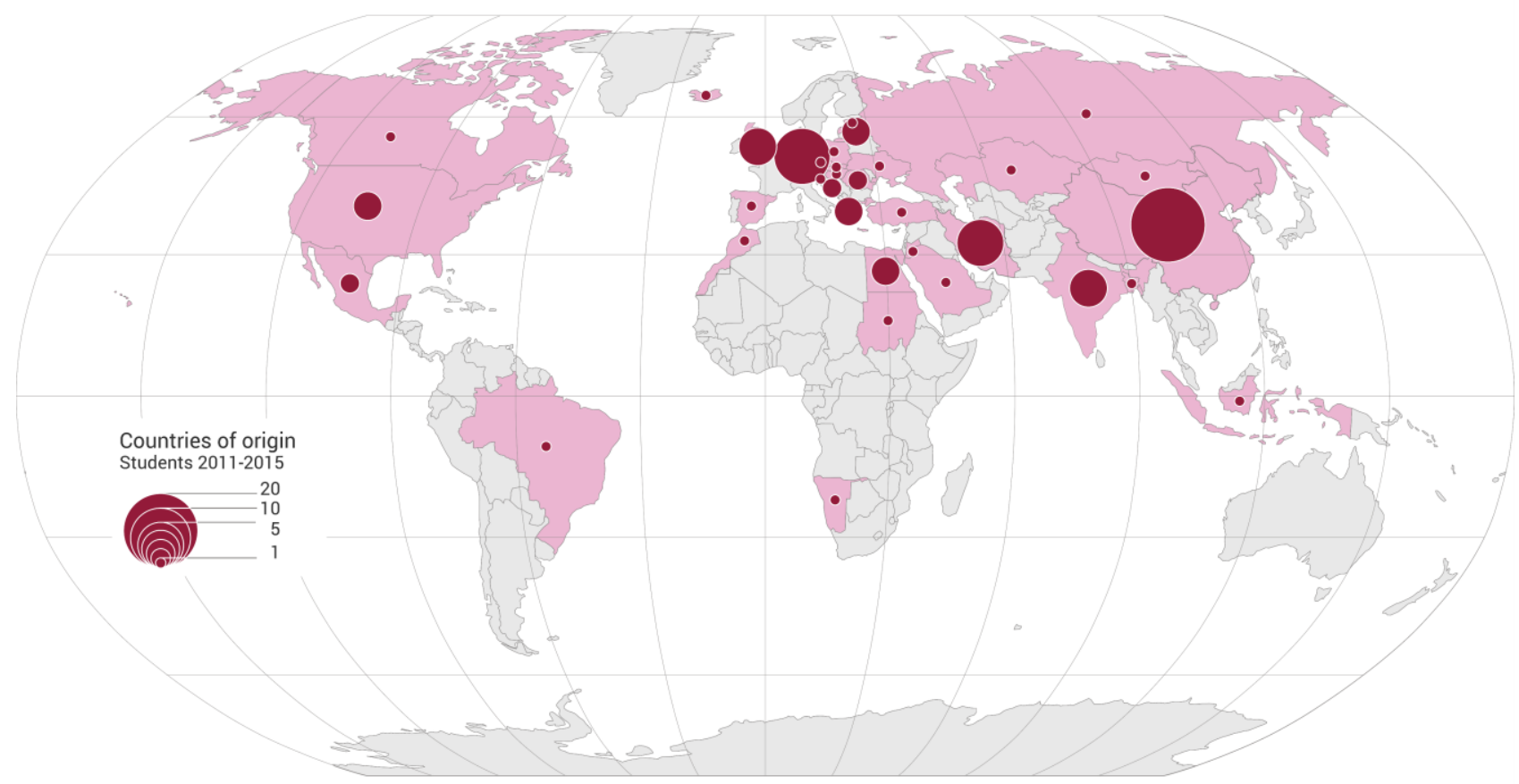

Figure 1: Countries of origin.

Most of the admitted students have a background in Geomatics, Geography, GIS and Remote Sensing (see Figure 2) and have a Bachelor degree (Figure 3).

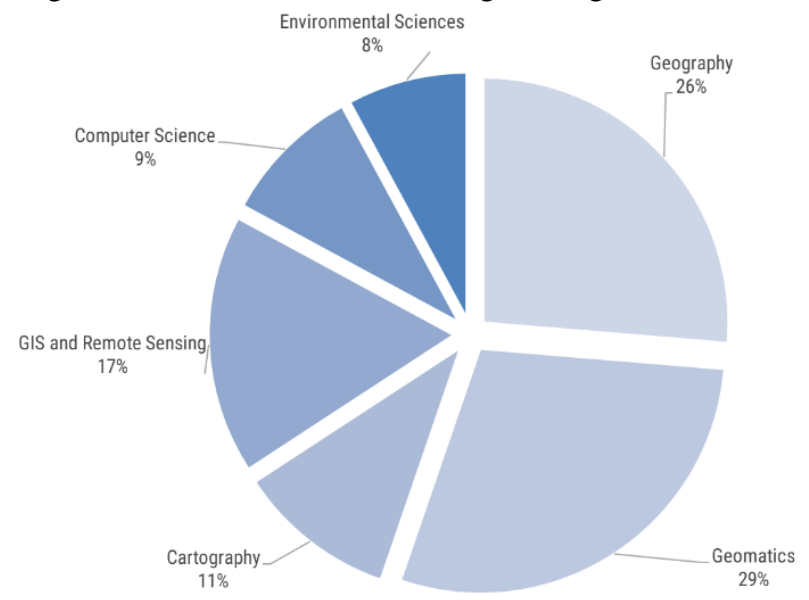

Figure 2. Students' background.



Figure 3. The highest university degrees of the candidates entering to the Cartography M.Sc. program.

\section{What knowledge do our graduates have?}

The program focuses on research questions in Cartography that were hardly studied before, such as spatio-temporal data integration for mapping purposes, location-based map services, personalized map design and user research. Students acquire in-depth expertise and competences in the entire field of cartography and gain fundamental knowledge in spatial data modelling, analysis and visualization of geographic information. Graduates are able to use modern theories, methods and procedures related to map production and map use in the sense of modern cartography, including geoinformatics, and are able to apply them professionally and economically. The graduates have the ability to capture, model, manage, analyze and visualize adequate spatial data with space, time and attribute information. They are in the position to handle databases and geographic information systems competently and to accomplish adequate graphic data processing for all kinds of user groups. Additionally, they are able to use all forms of publications and media such as print or electronic media. They can critically face up to social connecting factors and implications of various techniques and methods for processing and visualizing spatial data. Furthermore, a Master of Science in Cartography is in a position to strongly influence and shape cartography as an independent science with its own research and fount of knowledge, but also with an awareness of its distinct relations to the earth, information and communication sciences, having attained skills for the systematic analysis and synthesis relating the individual to the whole. Finally, graduates are able to develop their own study, research, work and management skills and are trained in oral and written communication. 
The consortium help students to recognize their preferable career path through entrepreneurial and research-oriented activities. Students have free access to career services and job fairs. Direct contact to future employees occurs through guest lectures, internships and theses topics. These projects involve real-world case studies, where students use critical, logical and cause/effect reasoning.

\section{What are employers looking for?}

Modern cartography needs experts with skills and knowledge in various backgrounds ranging from computer science to cognitive sciences. The added value of this specific program is that it is a pioneer for teaching the benefit of an interdisciplinary approach in a domain of growing relevance: Geo Spatial Information Management.

Potential employers look for candidates with both subject specific competences and soft skills (see Figure 4). The most demanded are: familiarity with GIS software, proficiency with handling geodata with databases and practical experiences in data visualization. At the same time analytical competences should be accompanied by excellent written and verbal communication, selfmotivation and a proactive attitude to work.



Figure 4. What are employers looking for in the Geo-Sector? (Data source: 145 Job descriptions published on www.digitalgeography.com/jobs between 20 April and 20 June 2018).

\section{Where are our graduates working?}

61 alumni from the first five intakes completed the questionnaire. The results from the survey indicate that cartography alumni work in an extensive range of organizations, in private business (about 40\%), public administration (about 20\%), and research institutions and universities (about 15\%). They have become consultants, product managers, cartographic designers, GIS experts, project managers, data analysts and scientists, web designers, software engineers and researchers. Figure 5 provides an overview of a sampling of organizations in which our alumni work.

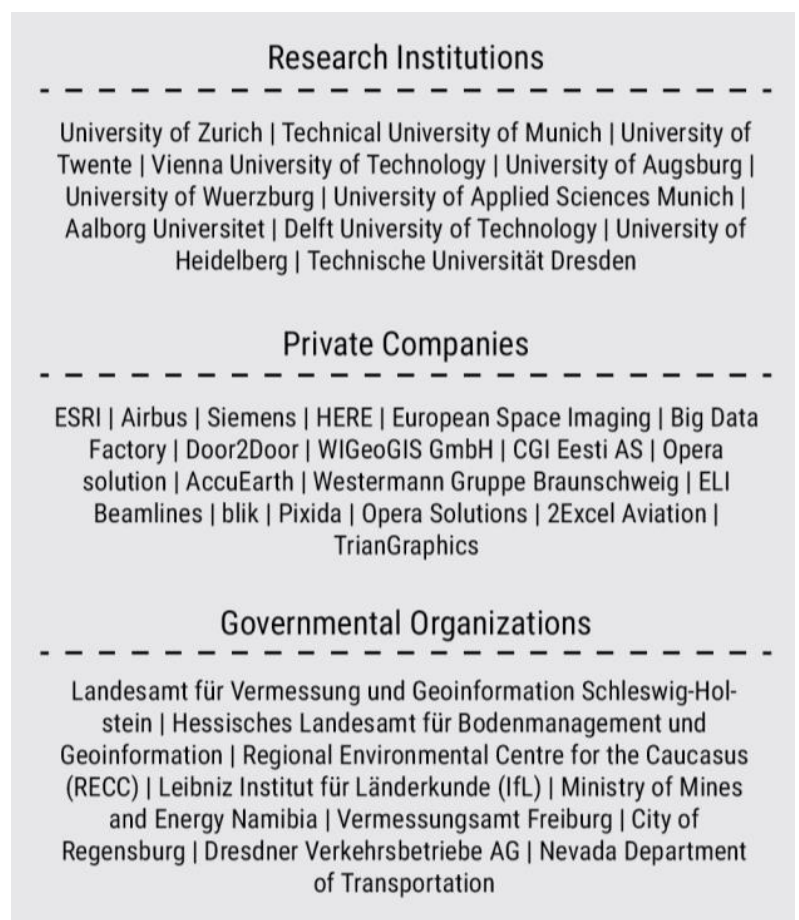

Figure 5. Employers of Cartography M.Sc. alumni (Effective: December 2018).

Cartography alumni are "global", but many of them stayed in one of the program countries after the graduation. Most of the students who have not returned to their home countries are located now in Germany, Switzerland and the Netherlands. Several students have taken positions elsewhere in Europe. Returning graduates have made impactful advances in their home country (Figure 6). 


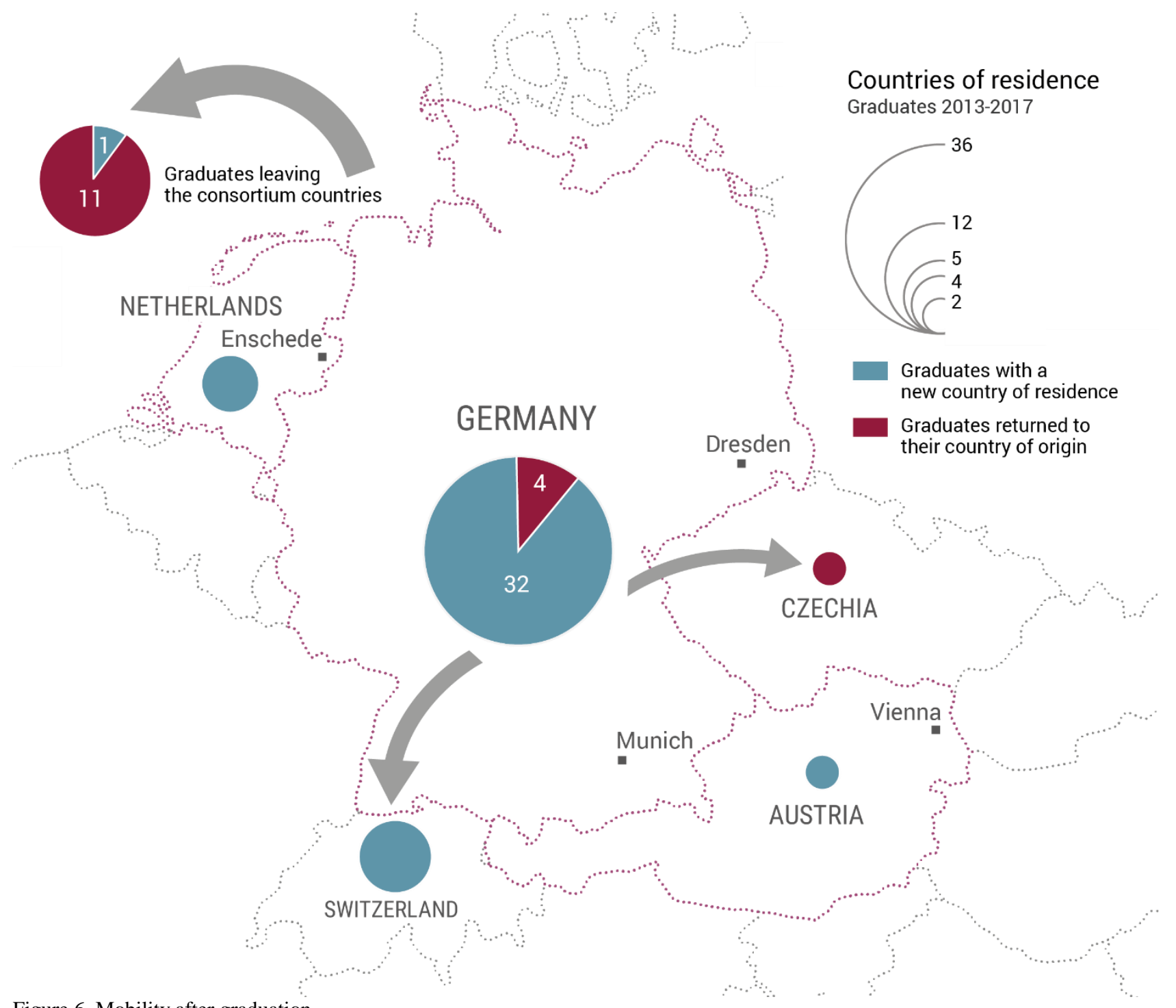

Figure 6. Mobility after graduation.

\section{What our graduates say about the program?}

The program curriculum challenges students with new situations. Most of the students need to instantly obtain knowledge and skills in the unknown fields, such as programming or image processing. At the same time students develop a deeper understanding of Cartography and Cartographic communication, what means they need to purposefully choose, present and justify their design steps in creation of various maps. Furthermore, they are expected to learn in the limited time the specifics of the university, new countries, culture and living environment.

In the survey we asked our graduates what competences have been proven to be the most relevant for their future careers. Beside technical skills such as programming, GIS and databases, they have mentioned several soft skills, which they obtained especially during the program. Students appreciated the possibility to creatively develop own ideas and put them into practice using the university resources, as mastering this workflow is required in their current occupation. Besides, students valued the experience of studying and working in different environments, as it shaped their ability to adapt to changes, organize their time and communicate with co-workers with different cultural background.

The obtained skills influence the way that alumni see their work prospects. They strongly believe that participation in the joint program have increased their chances to get a good occupation offer. They also can easily imagine working abroad at any point in the future, and developing their careers mostly in an international context. The following testimonials of alumni give further feedback on the relevancy of the program for the Geo-Sector and hints for future Cartography students:

\section{Mariana Danielova, ELI Beamlines Prague, Czechia}

"During the Cartography master program we were taught in cartographic fundamentals as well as in many other fields connected with cartography. Thus we knew what all the possible directions are and that we should not be afraid of something that we have hardly done before. For me 
personally I mostly valued the scientific visualization lectures, since I could never imagine that visualizations like that were possible. Next I enjoyed web cartography and mobile cartography lectures. I found out that I surprisingly like programming which together with visualizations became my dream job. In general, studying the MSc in Cartography was a perfect fit for my expectations and since graduation it has opened many doors for me in my professional career.

After graduation I worked 3 years for an international GIS company. Two years ago I decided to move closer towards my dream job which was meanwhile the biggest challenge for me because I was very new to programming. Therefore, I am currently happily working as a scientific data visualization specialist at the largest European laser center ELI Beamlines."

Maja Kalinic, University of Augsburg, Germany

"As someone who holds a degree in geodetic engineering, I was well aware of Cartography as a field of study, but also science and art. However, not until I became a student at International Master in Cartography, I realized its great potential and opportunities. For me personally, this was not only a second degree but also rather an intellectually stimulating place where I had the opportunity to gain knowledge and skills for my future (academic) career. Currently, I am doing my PhD at the Geoinformatics department, University of Augsburg. I deal with big traffic data and try to find best ways for detecting and predicting traffic congestions. I also teach and supervise students with their thesis research. I may say that this would not be possible or at least not comfortable as it is, if I have not learnt from the best while studying Cartography at four partner universities.

During the course, we all had to learn the fundamentals but we also had the chance to master many other useful skills through numerous elective courses. That is, actually, how I discovered my affection to programming (which I never done before) and big data handling. In addition, I learned how to utilize databases and geostatistics in problem-solving and decision-making. How to work on projects both solely and in team? I mastered these skills further by choosing the thesis topic that enabled me to learn even more and prepare for my PhD. Besides these skills, I wish to emphasize that this master gave me the chance to improve my scientific English, as well as scientific writing. This is something that is highly appreciated and needed in my academic career. Last, but not least, it gave me the opportunity to meet people from all over the world, enrich my understanding about the diversity of lifestyles, beliefs and values, and make friends for life."

\section{Katre Karja, CGI Eesti AS Tartu, Estonia}

"Studying in International Cartography was an inspiring and necessary phase in my life, as I found the program fit my interests, the lectures were motivating and I met so many wonderful people. The study gave me a solid foundation to spatial related and cartographic design related topics, as well it made it possible to get some experience with programming. Beside taken courses the work for my thesis allowed me to specialize further to the field of geovisualization with using Web technologies. This has given me the confident and suitable skills to fit into the team in which I currently work.

I work in a GIS team at an international company. At my current job I have various tasks for creating GIS applications. Mainly I work as a frontend developer to build the graphical interface of applications, and I also take care of design related tasks to create prototypes for the applications. Often in my work I need to deal with spatial databases. I think that the studies have given me an insight into the geo-world that supports finding creative solutions."

\section{Acknowledgements}

The authors would like to thank our program alumni for their active participation in the survey and insights they provided.

We are very grateful to the Education, Audiovisual and Culture Executive Agency of the European Union for cofunding the Cartography M.Sc. within the Erasmus+ initiative.

We would also like to show our gratitude to the associated partners of the program for their support in developing career and networking opportunities to the future job market of our students. 